\title{
A New Approach to Characterize Disordered Structures
}

\author{
J. Peinke, R. Friedrich, and A. Naert

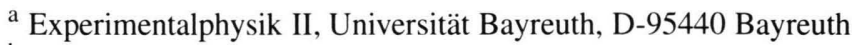 \\ ${ }^{\mathrm{b}}$ Institut für Theoretische Physik, Universität Stuttgart, D-70550 Stuttgart \\ ${ }^{c}$ R. I. E. C., Tohoku University, 2-1-1 Katahira, Aobaku, Sendai 980-77, Japan
}

Z. Naturforsch. 52 a, 588-592 (1997); received June 10, 1997

\begin{abstract}
A new application of the theory of Markov processes to the characterization of fractal scaling behavior is presented. We show under which condition distinct stochastic processes of a cascade lead to multifractal scaling behavior. We apply our method to the analysis of the statistical properties of the energy in turbulent fluid flow.
\end{abstract}

\section{Introduction}

There is an actual interest to characterize disordered structures $S$ exhibiting scaling behavior in terms of fractality or affinity, cf. [1]. The quantification of fractality is based on the introduction of a measurable quantity $Q(l)$ depending on a selected length scale. The $q$-th power of $Q(l)$, averaged over $S$, is investigated and it is examined whether there exists multiscaling behavior in the limiting case $l \rightarrow 0$ :

$$
\left\langle(Q(l))^{q}\right\rangle \sim l^{\zeta_{q}}
$$

Under this scaling assumption, scaling exponents $\zeta_{q}$ are deduced which serve as a characterization of the statistical properties of the disordered structure of $S$. If $\zeta_{q}$ is a nonlinear function of $q$ we say that $S$ is a multifractal or has multiaffine scaling properties [1].

For synthetically constructed complex structures like the Cantor-set or the Sierpinski-gasket (just to mention two well-known examples), the exponents $\zeta_{q}$ can be calculated explicitly. Problems commonly arise in the investigation of natural structures with the quality of this characterization method.

Another common method to characterize the statistical content of the disordered structure $S$ is the evaluation of correlations. Let $s(x)$ denote a functional value of $S$ at the location $x$ (for example, $x$ can be thought of as space variable but may also be considered as a time variable). Then $\left\langle s^{q_{1}}\left(x_{1}\right) s^{q_{2}}\left(x_{2}\right) \ldots s^{q_{n}}\left(x_{n}\right)\right\rangle$ is the general form of an

Reprint requests to Dr. J. Peinke, Fax: +49 921552621 , email: joachim.peinke@uni-bayreuth.de. $n$-point correlation. The knowledge of all $n$-point correlations can be regarded as a complete statistical characterization of $S$.

The multiscaling analysis and the correlation analysis is linked. Let us consider the definition $Q(l):=s(x+l)-s(x)$. Here $Q(l)$ is denoted as an increment. In this case the two-point-correlations $\left\langle s^{q_{1}}\left(x_{1}\right) s^{q_{2}}\left(x_{2}\right)\right\rangle=\left\langle s^{q_{1}}\left(x_{1}\right) s^{q_{2}}\left(x_{1}+l\right)\right\rangle$, where $l=$ $x_{2}-x_{1}$ are directly linked to $\left\langle(Q(l))^{q}\right\rangle$. In this case it is common to call $\left\langle(Q(l))^{q}\right\rangle$ the $q$-th order structure function.

\section{Evolution Equation for the Probability Distri- bution}

In this paper we want to present a new method to analyze disordered structures in a more general way. We will show that the two stochastic aspects just mentioned are incorporated in our approach. Starting from (1), we know that

$$
\left\langle(Q(l))^{q}\right\rangle=\int(Q(l))^{q} P(Q(l), l) \mathrm{d} Q(l) .
$$

Thus instead of evaluation the scaling behavior of (1) it is more general to evaluate the $l$-dependence of the probability density distribution $P(Q(l), l)$.

The essential point of our work is that we present a procedure which allows us to derive an evolution equation for the probability distribution $P(Q(l), l)$ with respect to the size parameter $l$ directly from experimental data. The first step consists in investigating how the quantity $Q(l)$ at one fixed point $x$ changes with $l$. (Also other constructions of $Q(l)$ may be used, like the midpoint construction: $Q(l):=$ 
$s(x+l / 2)-s(x-l / 2)$.) Form the data we can evaluate the $n$-dimensional probability density distributions

$$
p\left(Q\left(l_{1}\right), l_{1} ; Q\left(l_{2}\right), l_{2} ; \ldots ; Q\left(l_{n}\right), l_{n}\right)
$$

or respective conditional probability distributions

$$
p\left(Q\left(l_{n}\right), l_{n} ; \ldots ; Q\left(l_{r}\right), l_{r} \mid Q\left(l_{r-1}\right), l_{r-1} ; \ldots ; Q\left(l_{1}\right), l_{1}\right),
$$

where we use the convention $l_{i+1}<l_{i}$. Next we ask whether these $n$-dimensional distributions can be expressed by two-dimensional probability distributions. This is nothing else but investigating whether the statistics of $Q(l)$ corresponds to a Markov process. To give evidence that a Markov-process is related to our problem one should show that

$$
\begin{gathered}
p\left(Q\left(l_{n}\right), l_{n} ; \ldots ; Q\left(l_{r}\right), l_{r} \mid Q\left(l_{r-1}\right), l_{r-1} ; \ldots ; Q\left(l_{1}\right), l_{1}\right) \\
=p\left(Q\left(l_{n}\right), l_{n} ; \ldots ; Q\left(l_{r}\right), l_{r} \mid Q\left(l_{r-1}\right), l_{r-1}\right) .
\end{gathered}
$$

This is evidently an impossible task. However, as is well-known, a necessary condition for a process to be Markovian is the validity of the ChapmanKolmogorov equation [2]

$$
\begin{aligned}
& p\left(Q\left(l_{3}\right), l_{3} \mid Q\left(l_{1}\right), l_{1}\right)= \\
& \int p\left(Q\left(l_{3}\right), l_{3} \mid Q\left(l_{2}\right), l_{2}\right) p\left(Q\left(l_{2}\right), l_{2} \mid Q\left(l_{1}\right), l_{1}\right) \mathrm{d} Q\left(l_{2}\right) .
\end{aligned}
$$

The validity of this equations yields strong hints that the stochastic process is Markovian.

Form the Chapman-Kolmogorov equation an evolution equation for the conditional probabilities can be deduced, which is known as the Kramers-Moyal expansion

$$
\begin{aligned}
-\frac{\mathrm{d}}{\mathrm{d} l} & P(Q(l), l) \\
& =\sum_{k=1}^{\infty}\left[-\frac{\partial}{\partial Q(l)}\right]^{k} D^{(k)}(Q(l), l) P(Q(l), l) .
\end{aligned}
$$

Here, the Kramers-Moyal coefficients $D^{(k)}(Q(l), l)$ are defined by the following conditional moments:

$$
\begin{aligned}
& M^{(k)}(Q(l), l, \Delta l)=\frac{1}{\Delta l} \int \mathrm{d} Q(l-\Delta l) \\
& \cdot(Q(l-\Delta l)-Q(l))^{k} p(Q(l-\Delta l), l-\Delta l \mid Q(l), l) \\
& D^{(k)}(Q(l), l)=\lim _{\Delta l \rightarrow 0} \frac{1}{n !} M^{(k)}(Q(l), l, \Delta l)
\end{aligned}
$$

An important feature of the present approach is based on the fact that these Kramer-Moyal coefficients can actually be evaluated directly from the given data.

\section{Markov-process and Multifractality}

In the following we want to discuss some further implications based on well-known features of Markov-processes [2] which, however, have not yet been put into direct connection with the phenomenon of multifractality. (We would like to mention that the presented procedure may also be seen in analogy to the application of the Frobenius Peron operator for the determination of the natural measure of chaotic attractors [1].)

1. If $D^{(4)}=0$ Pawula's theorem implies that only the drift term $D^{(1)}$ and the diffusion term $D^{(2)}$ are non-zero. The Kramers-Moyal expansion (4) reduces to the Fokker-Planck equation

$$
\begin{aligned}
& -\frac{\mathrm{d}}{\mathrm{d} l} P(Q(l), l)=-\frac{\partial}{\partial Q(l)} D^{(1)}(Q(l), l) P(Q(l), l) \\
& +\left[\frac{\partial}{\partial Q(l)}\right]^{2} D^{(2)}(Q(l), l) P(Q(l), l) .
\end{aligned}
$$

2. From (4) and (7) the evolution equation for a single event $Q(l)$ at point $x$ in form of a Langevin equation can be derived [2]:

$$
-\frac{d}{d l} Q(l)=g(Q(l), l)+h(Q(l), l) \Gamma(l) .
$$

The function $g$ describes the deterministic evolution of $Q(l)$, whereas $h$ takes into account the stochastic fluctuations. In the case of the Fokker-Planck equation the functions $g$ and $h$ are given by $D^{(1)}$ and $D^{(2)}$, where $h \propto \sqrt{D^{(2)}}$. The noise term $\Gamma$ has the properties of $\delta$-correlated gaussian noise.

3. From (4), equations for the moments $\left\langle(Q(l))^{q}\right\rangle$ are obtained by multiplying (4) with $Q^{q}$ and successively integrating over $Q(l)$ [3]:

$$
-\frac{\mathrm{d}}{\mathrm{d} l}\left\langle(Q(l))^{q}\right\rangle=\sum_{n=1}^{q-1} \frac{q !}{(q-n) !}\left\langle D^{(n)} Q^{q-n}\right\rangle .
$$

4. If the Kramer-Moyal coefficients are

$$
D^{(n)} \sim \frac{d_{n} Q^{n}}{l}
$$


the scaling behavior of (1) is guaranteed and we obtain from (9)

$$
\frac{l}{\left\langle Q^{q}\right\rangle} \frac{\mathrm{d}}{\mathrm{d} l}\left\langle Q^{q}\right\rangle=-\sum_{n=1}^{q-1} \frac{q !}{(q-n) !} d_{n}=\zeta_{q} .
$$

For the simple case of a Fokker-Planck equation we see now that $D^{(1)}=d_{1} Q / l$ and $D^{(2)}=d_{2} Q^{2} / l$ implies multiscaling with $\zeta_{q}=d_{1} q-d_{2} q(q-1)$. The quadratic $Q$-dependence of $D^{(2)}$ corresponds to a purely multiplicative noise process, as we can see from the discussion of point 2 above.

5. For the case of the validity of a Fokker-Planck equation the stationary solution

$$
P(Q(l), l)=\frac{\text { const }}{\mathrm{D}^{(2)}} \exp \left(2 \int_{Q_{0}}^{Q(l)} \frac{D^{(1)}(y, l)}{D^{(2)}(y, l)} \mathrm{d} y\right)
$$

is known, where $Q_{0}$ is a constant. Taking the condition for scaling behavior of point four, (10), that the drift term is linear and the diffusion term is quadratic in $Q$, we find that the probability density distribution $P(Q(l), l)$ evaluated by $(12)$ is not normalizable. The stationary distribution is simply the $\delta$-function $\delta(Q(l))$. Inclusion of a small additive noise term yields a distribution which for large values of $Q(l)$ has a powerlaw form, which is a characteristic feature of a Lévy-distribution. In this case the moments $\left\langle Q^{q}\right\rangle$ diverge for higher $q$ values and thus the derivation of (9) becomes questionable [4]. An argument that the above mentioned results on $\left\langle Q^{q}\right\rangle$ still hold is that the power law tails of the distribution at infinity are only valid for the stationary solution. For an initial probability distribution at large scales $l$ it would take an infinite cascade (development with the evolution equation) to create such wings at infinity. Therefore, for any natural structure $S$, where typically scaling behavior is only found in a finite interval of scales $l$, we expect that these divergences do not affect the results discussed here.

6. We want to point out that having shown the Markovian properties any $n$-point statistics can be derived from the two-point statistics $p\left(Q\left(l_{2}\right), l_{2} \mid Q\left(l_{1}\right), l_{1}\right)$. In the case of the applicability of the Fokker-Planck equation the $n$-point statistics can be explicitly given by the knowledge of the two Kramer-Moyal coefficients $D^{(1)}$ and $D^{(2)}$ [2].

\section{Application to Experiments}

Finally, let us mention some verifications and applications of the method presented above:

For the case of fully developed turbulence, a major challenge consists in explaining the statistics of velocity increments $V(l)$, cf. [5]. Typically the increments of the velocity component in the direction of the distance vector are taken. Investigation of experimental data has recently shown that the statistics of the velocity increments can be characterized by a Fokker-Planck equation with $D^{(1)}=\gamma V(l) / l$ and $D^{(2)}=a(l)+b(V(l))^{2} / l$, [6]. Here, $\gamma$ turns out to be approximately $-1 / 3$, which is clearly related with the scaling behavior of the second moments, which scale like $\left\langle V(l)^{2}\right\rangle \approx l^{\zeta_{2}}, \zeta_{2} \approx 2 / 3$.

Here we want to report on further new findings on the disorder in turbulence. It is of central interest to determine the statistics of the energy dissipation on different length scales. Following the suggestion of Kolmogorov and Obukhov, we evaluate the energy dissipation at scale $l$ according to [7]:

$$
\epsilon_{l}(x)=\frac{15 \nu}{l} \int_{x-l / 2}^{x+l / 2}\left(\frac{\mathrm{d} v}{\mathrm{~d} x^{\prime}}\right)^{2} \mathrm{~d} x^{\prime},
$$

where $\nu$ denotes the kinematic viscosity. It has been postulated that nongaussian statistics in the velocity increments is due to a log-normal distribution of $\epsilon$. We take $\log (\epsilon)$ as our quantity $Q$. The variance of $\log (\epsilon)$ was assumed to be logarithmic in the length scale. We have already verified the Chapman-Kolmogorov equation [8]. Next we have evaluated the KramersMoyal coefficients, and we saw that the fourth coefficient is close to zero.

Figure 1 presents the first two Kramers-Moyal coefficients, $D^{(1)}(Q, l)$ and $D^{(2)}(Q, l)$. Both coefficients have been evaluated for several scales covering the whole inertial range. (The inertial range in turbulence denotes the range of length scales where scaling behavior is expected to occur. This range is bounded by a large length scale $L$ dominated by the boundary conditions, like the size of the flow, and the viscous or Kolmogorov length scale $\eta$, where dissipation dominates the flow.) For convenience we have used in our evaluation a logarithmic length scale $l=\ln r$, which corresponds in evaluation $r D^{(n)}$ in a linear scale. In the following, $r$ denotes the linear scale. (Note that these Kramers-Moyal coefficients presented are obtained for a finite scale ratio of $r_{1} / r_{2}=1.02$, just 

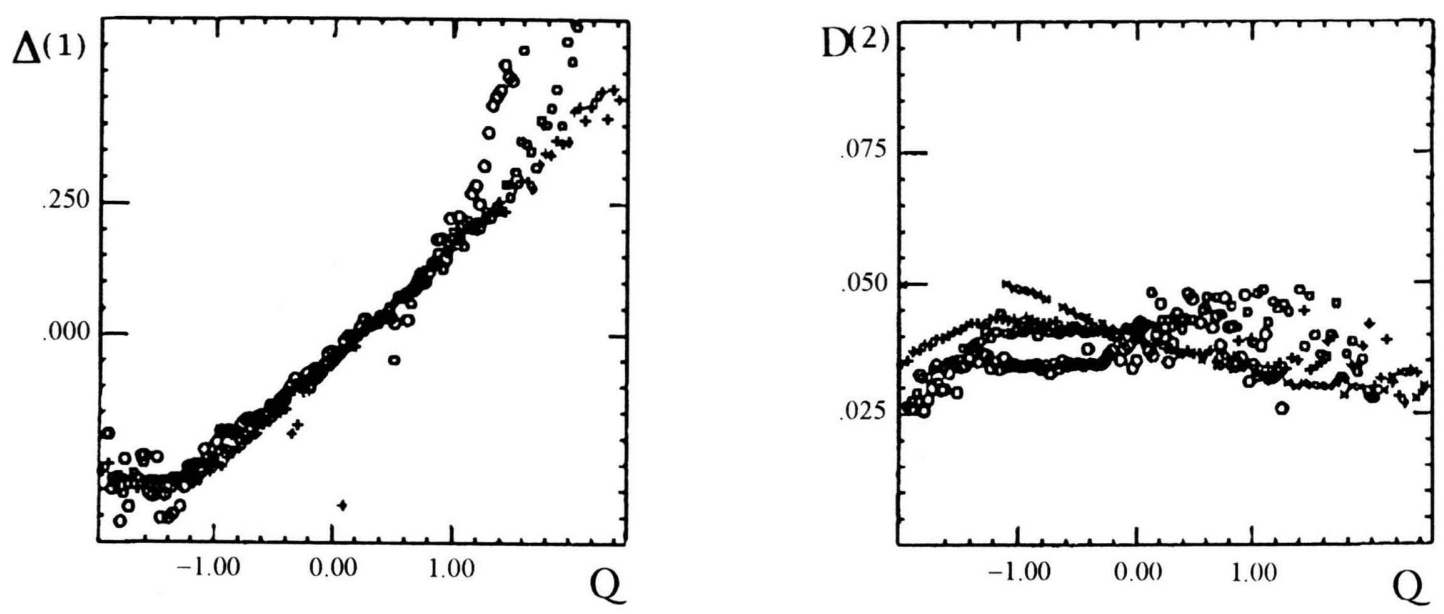

Fig. 1. Drift and diffusion coefficients, $\Delta^{(1)}=D^{(1)}-F(l)$ and $D^{(2)}$ for the values of the scale $r / \eta=400,200,100,50$, $\eta$ being the Kolmogorov viscous scale. These coefficients were evaluated from experimental data of a free jet with a Reynolds number $R_{\lambda}$ of 328 [9].

below which the resolution of the velocity is not sufficient any more.)

To make the drift terms coincide an $l$-dependent additive term $F(l)$ has been used. The occurence of this additive term can be justified. In fact it is determined by the constraint of conservation of mean energy.

In the central region, the drift coefficient is linear in $\log \left(\epsilon_{r}\right)=Q$ and the diffusion coefficient is constant. Furthermore, the slope of $D^{(1)}$ and the value of $D^{(2)}$ are both scale invariant in the inertial range:

$$
\begin{aligned}
& D^{(1)}(Q)=\gamma(Q-\langle Q\rangle)+F(l) \\
& D^{(2)}(Q)=D
\end{aligned}
$$

with $\gamma \approx 0.2$ and $D \approx 0.03$. Thus, we can summarize that our empirical results show that the statistics of the quantity $\log \left(\epsilon_{r}\right)$ is determined by a Fokker-Planck equation with a linear drift and a constant diffusion coefficient. Such a Fokker-Planck equation is related to an Ornstein-Uhlenbeck process. The positive slope of $D^{(1)}$ indicates that there is no stationary probability distribution [2]. Nevertheless, the following conclusions on the evolution of the probability distributions can be drawn.

As initial condition at the integral scale $L$ we take a sharp distribution:

$$
P\left(Q_{L}\right)=\delta\left(Q_{L}-\log \langle\epsilon\rangle\right)
$$

where $\langle\epsilon\rangle$ denotes the mean dissipation. The conditional distribution function for $r<L, P(Q(r) \mid Q(L))$, is then Gaussian:

$$
P(Q(r) \mid Q(L))=\frac{1}{\Lambda \sqrt{2 \pi}} e^{-(Q(r)-\langle Q(r)\rangle)^{2} / 2 \Lambda^{2}},
$$

where the mean and the variance are given by [2]

$$
\begin{aligned}
\langle Q(r)\rangle & =\langle Q(L)\rangle-\Lambda^{2} / 2, \\
\Lambda^{2} & =\frac{D}{2 \gamma}\left(\left(\frac{r}{L}\right)^{-2 \gamma}-1\right) .
\end{aligned}
$$

In the range of scales $r \ll L, \Lambda^{2}$ can be written as a power law:

$$
\Lambda^{2}=\frac{D}{\gamma}\left(\frac{r}{L}\right)^{-2 \gamma}
$$

Such a dependence has been predicted by Castaing [10] for a quantity $\lambda^{2}$ proportional to $\Lambda^{2}$. For large scales $r, \Lambda^{2}$ becomes small and may be approximated by a logarithmic law leading to scaling behavior in the velocity increments. 


\section{Summary}

To summarize, we have presented a new method to analyze the statistical content of a disordered structure. We have shown how this approach is related to multifractal scaling. Furthermore, is has been shown that this method can be applied to experimental data; therefore we have presented the analysis of the energy statistics in turbulence. From this we found that the statistics are very close to a simple well-known stochastic process, namely the Ornstein-Uhlenbeck process. If one verified that the disorder of a system can be attributed to a stochastic process, one has the opportunity to use many results explaining details of the disorder. Thus we saw for the energy cascade in turbulence that the positive slope of the drift term leads to the nonstationary spreading of the width of the probability distributions. This behavior causes the often discussed intermittency effects in turbulence directly. These intermittency effects are usually discussed in terms of multifractal scaling, but up to now no satisfying conclusion could be drawn of the multifractal scaling ansatz. With the analysis presented here, we hope to have given some new insight into the statistics of the energy cascade in turbulence.

\section{Acknowledgements}

Helpful discussions with Martin Greiner, Peter Lipa, and the members of ENGADYN are acknowledged. JP acknowledges financial support by the Deutsche Forschungsgemeinschaft. The paper was presented at 6th Annual Meeting of ENGADYN, Tüchersfeld 1996.
[1] R. Badii and A. Politi, Complexity, Cambridge University Press, Cambridge 1997; - B. Mandelbrot, The Fractal Geometry of Nature, Freeman, San Francisco 1982; - K. J. Falconer, Fractal Geometry, Mathematical Foundations and Applications, John Wiley, Chichester 1990; - J. Feder, Fractals, Plenum Press, New York 1988); - T. Vicsek, Fractal Growth Phenomena, World Scientific, Singapore 1992.

[2] P. Hänggi and H. Thomas, Physics Reports 88, 207 (1982); - N. G. van Kampen, Stochastic Processes in Physics and Chemistry, Elsevier, Amsterdam 1990; - H. Risken, The Fokker-Planck Equation, SpringerVerlag Berlin 1984.

[3] For the integartion it is assumed that the probability distributions $P(Q(l), l)$ decay at infinity faster than any power of $Q$.
[4] private communication with Peter Lipa.

[5] K. R. Sreenivasan and R. A. Antonia, Annu. Rev. Fluid Mech. 29, 435 (1997).

[6] R. Friedrich and J. Peinke, Physica D 102, 147 (1997); - R. Friedrich and J. Peinke, Phys. Rev. Lett. 78, 863 (1997).

[7] A. M. Obukhov, J. Fluid Mech. 13, 77 (1962); A. N. Kolmogorov, J. Fluid Mech. 13, 82 (1962).

[8] A. Naert, R. Friedrich, and J. Peinke, A Stochastic Equation for the Energy Cascade in Turbulence, Phys. Rev. E (in press).

[9] B. Chabaud, A. Naert, J. Peinke, F. Chillà, B. Castaing, and B. Hébral, Phys. Rev. Lett., 73, 3227 (1994).

[10] B. Castaing, Y. Gagne, and E. Hopfinger, Physica D 46, 177, (1990). 